\title{
Parenteral nutrition for symptom control in a patient with pancreatic cancer and generalized oedema - a case report
}

\author{
R. Simanek ${ }^{1}$, A. Henry'2, D. Weixler ${ }^{3}$, B. Hammerl-Ferrari' ${ }^{1}$, K. Geissler ${ }^{1}$, H. Watzke ${ }^{4}$ \\ 1Hietzing Hospital, 5th Medical Department with Oncology and Palliative Care Unit, Vienna, Austria \\ ${ }^{2}$ Caritas Socialis Hospice Rennweg, Palliative Care Unit, Vienna, Austria \\ 3Horn Hospital, Department of Anaesthesiology and Intensive Care, Palliative Support Team, Horn, Austria \\ ${ }^{4}$ Medical University of Vienna, Department of Internal Medicine 1, Palliative Care Unit, Vienna, Austria
}

Received 13 February 2012; accepted 6 March 2012

The effect of parenteral nutrition (PN) in advanced cancer patients is exemplified at the clinical case of a male, 82-year old patient. Methods: Case report and short survey of the current literature. Results: In our patient with locally advanced pancreatic cancer, inflammatory state and recurrent ascites/ oedema due to an anorexia-cachexia-syndrome, persistent symptom control could be reached by antiinflammatory treatment and supplemental PN until his death.

Keywords: Parenteral nutrition, cancer cachexia, symptom control, oedema, palliative care.

\section{Introduction}

The role of continuous parenteral nutrition (PN) in patients with incurable cancer is controversial. In cancer patients malnutrition was identified as a risk factor for a number of clinical problems within the last years [1], but recent guidelines do not generally recommend $\mathrm{PN}$ in patients with incurable malignancies without bowel obstruction [2], mostly due to a new understanding of the complex mechanisms of cachexia that are weakly related with anorexia and decreased nutritional intake [3].

Proinflammatory cytokines cause an ongoing loss of skeletal muscle mass, which is not fully reversible by conventional nutritional support leading to progressive functional impairment and involuntary weight loss. Recently, a definition and classification of cachexia in cancer patients was published, mainly focusing on three domains: percentage of weight loss, body mass index and sarcopenia. Furthermore, various stages (precachexia to cachexia to refractory cachexia) were described [4].

Therapeutic approaches for the treatment of the cancer-related anorexia-cachexia syndrome are anti-inflamma-

Correspondence: Ralph Simanek, MD, Hietzing Hospital, 5th Medical Department with Oncology and Palliative Care Unit,

Wolkersbergerstraße 1, 1130 Vienna, Austria.

E-mail: ralph.simanek@wienkav.at tory treatment and anti-cancer treatment (due to the fact that the tumour itself can act as a "catabolic driver"). Other effective medical treatments seem to be progestagens, corticosteroids, omega-3 fatty acids and cannabinoids, next to other substances with a lower level of evidence [5]. Basic conditions for a regular food intake should be kept in mind, secondary types of the anorexia-cachexia syndrome as impaired oral intake, impaired gastrointestinal absorption or infections with an additional catabolic state attended [6]. Additionally, nutritional recommendations for the management of sarcopenia have been published, highlighting the role of exercise in combination with adequate protein and energy intake [7].

\section{Clinical case}

In our patient (male, 82 yrs.) locally advanced pancreatic cancer (adenocarcinoma, histological grading II) was diagnosed after the patient had observed a painless ikterus. Following endoscopic retrograde cholangiopancreatography (ERCP), papillotomy and plastic-stent implantation, three cycles of gemcitabine and oxaliplatin were administered. After ERCP hepatic abscesses were observed, resulting in longer-lasting stops between the single chemotherapeutic cycles due to recurrent episodes of fever. Three months after diagnosis increasing cholestasis led to another ERCP with subsequent wall stent implantation. One month later computerized tomography showed increasing hepatic abscesses. Streptococcus mitis and Hafnia alvei were diagnosed in microbiological analysis, and antibiotic treatment with amoxicillin and clavulanic acid was started. An ascites puncture, performed one day before admission at our department, revealed 9,000 $\mathrm{ml}$ of ascites. Peritoneal carcinosis has never been shown in computerized tomography.

At the time of admission at our palliative care department 5 months after diagnosis, the patient presented generalized oedema, ascites, weakness and anorexia. Karnofsky index was $30 \%$, weight 70 kilograms (kg) and body mass index (BMI) 23.4. A weight loss of $8 \mathrm{~kg}(11 \%)$ within 6 months was reported by the patient. Laboratory parameters showed increased inflammation parameters and decreased prealbu- 
Tab. 1: Course of laboratory parameters

during hospital stay

\begin{tabular}{llll} 
Time (day of hospital stay) & 1 & 13 & 18 \\
\hline Leukocytes (G./l) & 11.5 & 7.81 & 5.99 \\
\hline C-reactive protein (mg/l) & 93.6 & 15.6 & 9.0 \\
\hline Prealbumin (mg/l) & 50 & 100 & n.a. \\
\hline Albumin (g/l) & 27 & 35 & n.a. \\
\hline Total protein (g/l) & 64 & 74 & n.a. \\
\hline Cholinesterase (U/l) & 921 & n.a. & n.a. \\
\hline n.a. not assessed. & & & \\
\hline
\end{tabular}

min, albumin and cholinesterase (Table 1). When abdominal sonography was performed, it showed the abscess cavity filled with air and only a small amount of fluid. Therefore, drainage of the abscess was not indicated. After port-a-cath implantation, parenteral antibiotic treatment and $\mathrm{PN}$ with a three-chamber bag were started (830 kilo-calories (kcal) day $1-5 ; 1100 \mathrm{kcal}$ from day 6$)$. Furthermore, the patient received oral enteral nutrition supplements ( $200 \mathrm{kcal}$ per day) in addition to his regular meals. Human albumin $(20 \%, 100$ millilitres $(\mathrm{ml})$ ) was added for 2 weeks, and spironolactone (100 milligrams (mg) day 1-6; $50 \mathrm{mg}$ from day 7) was administered. On the 6th day during the patient's stay, a new ascites puncture was performed and 7,000 $\mathrm{ml}$ was drained.

It was evident that the patient had problems to retain facts in short-term memory. After a mini mental state examination, indicating 21 points, anti-dementia treatment was started. The patient began physical examinations instructed by a physiotherapist.

All these measures led to an improvement in the abovementioned laboratory parameters, a weight loss of $14 \mathrm{~kg}$ due to reduced oedema and increased mobility. There was no need for another ascites puncture. The patient was discharged after 19 days with a weight of $56 \mathrm{~kg}$ (BMI 18.7), decreased inflammatory parameters, a slightly increased prealbumin and albumin (Table 1) and a Karnofsky index of $60 \%$, and stayed at home with parenteral nutrition and continuous orally admitted antibiotic treatment until his death 4 months later. No episodes of fever, oedema or ascites occurred after discharge. Continuous antibiotic treatment and PN combined with physiotherapy resulted in an adequate persisting symptom control in our patient.

\section{Discussion}

The reason for starting and maintaining PN in our patient was to guarantee a high calorie supply next to oral nutrition supplements to treat hypoalbuminemia (caused by malnutrition next to inflammation), which seems to have played an essential role in our patient's symptomatology. Malnutrition and subsequent protein deficiency were probably hindered by dementia (a secondary cause for cachexia?), causing irregular eating habits. Additionally, in the absence of peritoneal carcinosis, the genesis of ascites and generalized oedema might be caused due the inflammatory state and a subsequent, reversible capillary leak syndrome due to hepatic abscesses, leading to continuous antibiotic treatment.
Weight measurement was problematic due to generalized oedema; presumably the initially measured BMI was incorrectly high. Considering this problem, all criteria for cachexia (weight loss greater than 5\%, BMI lower than 20, sarcopenia, reduced food intake and systemic inflammation) were found in our patient.

A recently published study found out that home $\mathrm{PN}$ is used in about $11 \%$ of cancer patients enrolled in palliative home care services, and contrary to the existing guidelines, it is also used to supplement oral intake even in the absence of a non-functioning gastrointestinal tract [8]. PN was shown to improve mean body mass index (BMI), quality of life and survival in patients with advanced cancer and $\mathrm{PN}$ combined with oral enteral nutrition supplement compared to patients with oral nutrition supplement alone [8]. Similar findings were presented in recently published studies investigating the role of additional PN in patients with advanced pancreatic cancer [9]. Further study results on PN in pancreatic cancer patients are outstanding [10].

Recently it was shown that improvement in physical activity might be a variable to measure treatment effects of anticachexia therapy in cancer patients [11]. Our efforts resulted in an increased patient's mobility and an inclined Karnofsky index, probably caused by the improved nutritional state, and could be an advice for a slowed sarcopenia.

In the light of new findings on pathophysiological changes in cachectic cancer patients on the one hand and the large amount of $\mathrm{PN}$ products on the other hand, further studies focusing on the use of $\mathrm{PN}$ in this very special patient group are necessary. Recently a study investigating indications, goals/outcomes and practices of PN in patients with malignant bowel obstruction was presented on the 6th "Cachexia Conference" in Milano 2011 [12]. Studies like this might be helpful for the development of new guidelines supporting the decision making on the use of PN in patients with advanced cancer.

\section{Conflict of interest}

The authors declare that there is no conflict of interest.

\section{References}

[1] Dewys WD, Begg C, Lavin PT, et al. Prognostic effect of weight loss prior to chemotherapy in cancer patients. Eastern Cooperative Oncology Group. Am J Med, 69(4): 491-7, 1980.

[2] Bozzetti F, Arends J, Lundholm K, Micklewright A, Zurcher G, Muscaritoli M. ESPEN Guidelines on Parenteral Nutrition: non-surgical oncology. Clinical nutrition (Edinburgh, Scotland), 28(4): 445-54, 2009.

[3] Blum D, Omlin A, Baracos VE, et al. Cancer cachexia: a systematic literature review of items and domains associated with involuntary weight loss in cancer. Critical reviews in oncology/hematology, 80(1): 114-44, 2011.

[4] Fearon K, Strasser F, Anker SD, et al. Definition and classification of cancer cachexia: an international consensus. The lancet oncology, 12(5): 489-95, 2011.

[5] Mantovani G, Madeddu C. Cancer cachexia: medical management. Support Care Cancer, 18(1): 1-9, 2010.

[6] Strasser F. Eating-related disorders in patients with advanced cancer. Support Care Cancer, 11(1): 11-20, 2003.

[7] Morley JE, Argiles JM, Evans WJ, et al. Nutritional recommendations for the management of sarcopenia. J Am Med Dir Assoc, 11(6): 391-6, Review.

[8] Orrevall Y, Tishelman C, Permert J, Cederholm T. The use of artificial nutrition among cancer patients enrolled in palliative home care services. Palliat Med, 23(6): 556-64, 2009. 
[9] Pelzer U, Arnold D, Govercin M, et al. Parenteral nutrition support for patients with pancreatic cancer. Results of a phase II study. BMC Cancer, 10: 86.

[10] Marten A, Wente MN, Ose J, et al. An open label randomized multicentre phase IIIb trial comparing parenteral substitution versus best supportive nutritional care in subjects with pancreatic adenocarcinoma receiving 5-FU plus oxaliplatin as 2nd or higher line chemotherapy regarding clinical benefit - PANUSCO. BMC Cancer, 9: 412, 2009.
[11] Mantovani G, Madeddu C, Serpe R. Improvement of physical activity as an alternative objective variable to measure treatment effects of anticachexia therapy in cancer patients. Current opinion in supportive and palliative care, 4(4): 259-65.

[12] Nestor K. Parenteral nutrition in patients with cancer cachexia and bowel obstruction: international survey on indications, outcomes and practices; 6th Cachexia Conference, Abstract (6-08) Milano 2011. 